\title{
Correction of dopant concentration fluctuation effects in silicon drift detectors.
}

\author{
D. Nouais ${ }^{\mathrm{a}, 1}$, M. Bondila ${ }^{\mathrm{b}}, \mathrm{V}$. Bonvicini ${ }^{\mathrm{c}}$, P. Cerello ${ }^{\mathrm{a}}$, \\ E. Crescio ${ }^{\mathrm{a}}, \mathrm{P}$. Giubellino ${ }^{\mathrm{a}}$, R. Hernández-Montoya ${ }^{\mathrm{a}, 2}$, \\ A. Kolojvari ${ }^{\mathrm{a}, 3}$, L.M. Montaño ${ }^{\mathrm{a}, 2}$, B.S. Nilsen ${ }^{\mathrm{d}}$, C. Piemonte ${ }^{\mathrm{c}}$, \\ A. Rashevsky ${ }^{\mathrm{c}}, \mathrm{F}$. Tosello ${ }^{\mathrm{a}}, \mathrm{A}$. Vacchi ${ }^{\mathrm{c}}, \mathrm{R}$. Wheadon ${ }^{\mathrm{a}}$, \\ for the ALICE collaboration. \\ ${ }^{a}$ INFN Sezione di Torino, Italy \\ b Jyväskylä University, Finland \\ ${ }^{\mathrm{c}}$ INFN Sezione di Trieste, Italy \\ d The Ohio State University, USA
}

\begin{abstract}
Dopant fluctuations in silicon wafers are responsible for systematic errors in the determination of the particle crossing point in silicon drift detectors. In this paper we report on the first large scale measurement of this effect by means of a particle beam. A significant improvement of the anodic resolution has been obtained by correcting for these systematic deviations.
\end{abstract}

Silicon drift detectors (SDDs) have been chosen to equip two layers of the inner tracking system of the ALICE[1,2] experiment at LHC due to their very good spatial precision and capability of unambiguous two-dimensional position determination in high multiplicity environments. In an SDD, under the effect of an applied electric field, the electrons liberated by the passage of a particle drift along a direction parallel to the surface of the wafer to an array of collection anodes perpendicular to the drift direction. The drift velocity is proportional to the electric field $E$ and to the electron mobility $\mu_{e}: V=\mu_{e} E$. In this way, the distance of the crossing point from the anodes is determined by the measurement of the drift time. The second coordinate is obtained from the centroid of the charge distribution across the anodes.

1 Corresponding author. E-mail: nouais@to.infn.it

2 also at CINVESTAV Mexico City, Mexico

3 also at St.Petersburg University, St. Petersburg, Russia

Preprint submitted to Elsevier Preprint

31 May 2000 
To reach the spatial precision required in ALICE (about $30 \mu \mathrm{m}$ along the two axes $[1,2])$, it is necessary either to have a drift field with an excellent uniformity over all the sensitive region of the detector, or to correct for the systematic errors caused by its non-uniformity. A non-uniformity of the field along the drift direction alters the proportionality between the drift time of the electron cloud and the distance of the crossing point of the particle from the anodes. This introduces an uncertainty on the drift axis coordinate. On the other hand, the presence of a parasitic transversal electric field in the wafer induces deviations of the electron trajectories from the ideal linear path as illustrated in fig. 1. These deviations are responsible for systematic errors on the anodic coordinate. Such transversal deviations have been studied on a

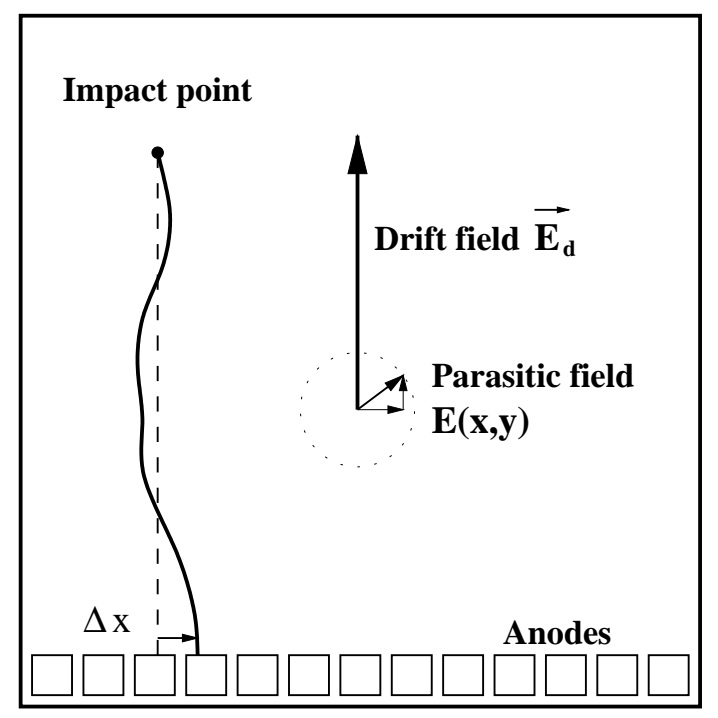

Fig. 1. Sketch of an electron trajectory in a SDD in presence of a parasitic field superimposed to the ideal drift field.

small area of a SDD, constructed on a floating zone wafer by using an infrared laser $[3,4]$.

In this paper, we present the first large scale study of these deviations, which we performed on an ALICE-D1 SDD prototype, produced on a $3 \mathrm{k} \Omega \mathrm{cm} 5$ " $n$-type neutron transmutation doped (NTD) silicon wafer [2]. The NTD technique has been chosen for its good uniformity of doping concentration. The detector active region is divided centrally into two separate zones each with a $35 \mathrm{~mm}$ maximum drift path read out at the edge of the detector by 256 anodes with a pitch of $294 \mu \mathrm{m}$. A detailed description of the ALICE SDD design is given in [5].

The results have been obtained from the data analysis of a beam test performed in May 1999, with a $375 \mathrm{GeV} / \mathrm{c}$ pion beam of the SPS. The SDD, operated at a drift field of $583 \mathrm{~V} / \mathrm{cm}$, was placed on the beam trajectory between two microstrip telescopes which allow to predict the particle impact 
point on the SDD with a precision of $5 \mu \mathrm{m}$ r.m.s. The detector was read out using the OLA chip [6], specially developed for this type of detector. An area of about $19 \times 35 \mathrm{~mm}^{2}$, corresponding to 64 anodes, has been studied.

This area has been divided in rectangles of $1 \times 0.3 \mathrm{~mm}^{2}$. For each rectangle, we built the residual distributions between the anodic coordinate measured by the SDD and the reference anode axis coordinate measured by the telescopes. The centroid of such local distribution is represented in fig. 2 as a function of the position of the rectangle center.

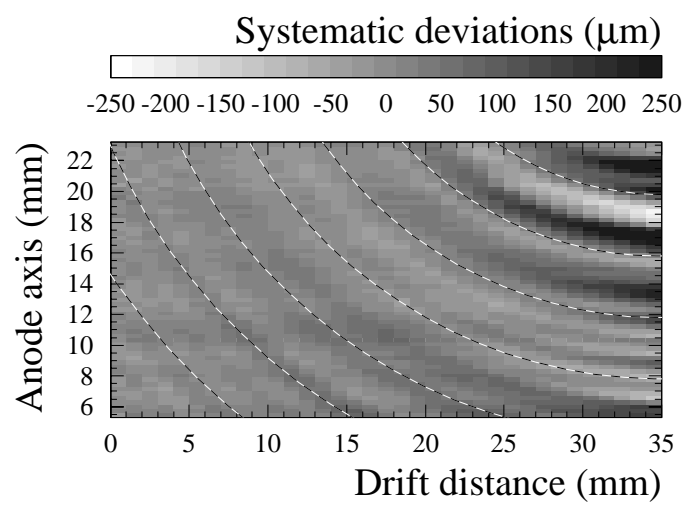

Fig. 2. Systematic deviations of the anodic coordinate of the electron cloud centroid with respect to the reference position of the crossing point of the particle, as a function of the drift distance and the anodic coordinate. The dashed arcs, centred on the middle of the silicon wafer, are plotted to allow an eye estimate of the central symmetry of the observed deviations.

Circular structures centred on the middle of the wafer (corresponding to the centre of the detector) are clearly observed in this plot. This effect can be attributed to the radial dependence of the dopant concentration fluctuations [7]. A simple numerical calculation of electron trajectories has been performed in order to evaluate the amplitude of the fluctuations needed to explain such deviations. A radial sinusoidal dopant concentration variation with a $4 \mathrm{~mm}$ spatial periodicity and a $2 \%$ amplitude has been simulated, with the result illustrated in fig. 3. This preliminary result reproduces quite well the qualitative features and the amplitude of the deviations. In both the data and the simulations, the amplitude of the deviations can be seen to increase with the drift distance. This can be explained by the fact that the parasitic electric field produced by the dopant fluctuations is perpendicular to the drift field at the maximum drift distance, and hence the deviation from the linear trajectory is higher in this region. The amplitude of the dopant concentration variations is of the same order of magnitude of those observed on identical wafers $[8,9]$. Since the maximum systematic deviations have been measured to be of about $300 \mu \mathrm{m}$, it is clearly indispensable to correct for this effect.

The anodic resolution is defined as the r.m.s. of the residual distribution be- 


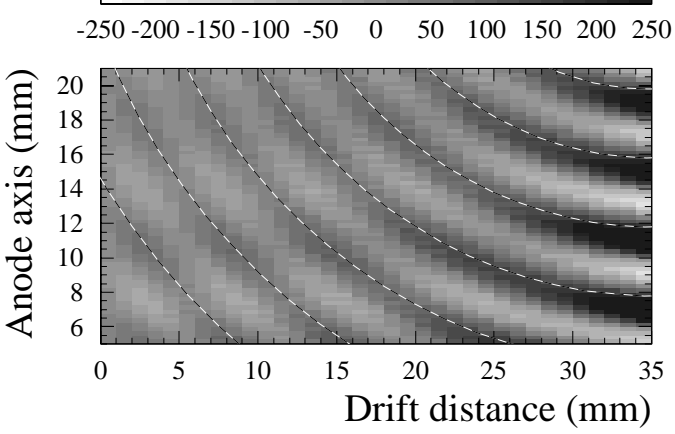

Fig. 3. Systematic errors on the anodic coordinate as a function of the drift distance predicted by simulating the electron trajectories in a drift electric field with perturbations caused by dopant concentration fluctuations. The dopant concentration has a radial sinusoidal dependence with a $4 \mathrm{~mm}$ spatial periodicity and a $2 \%$ amplitude.

tween the anode axis coordinates measured by the SDD and by the microstrip telescopes. In fig. 4, the resolution as a function of the drift distance with and without corrections of the systematic deviations measured using the beam test data are compared.

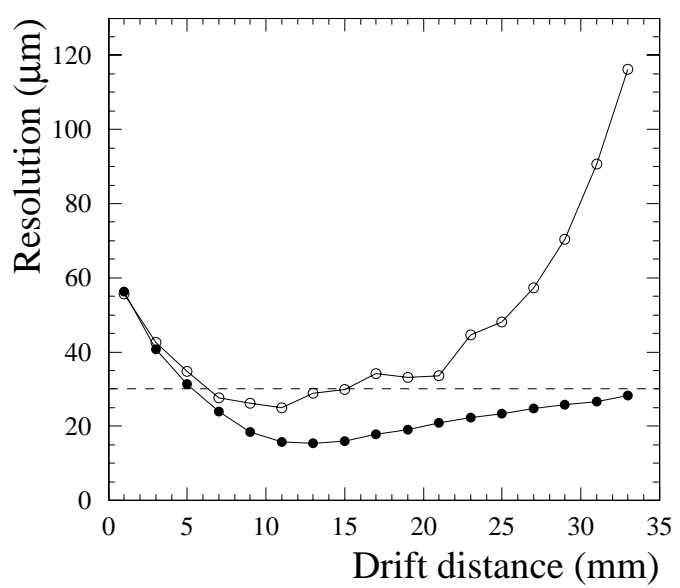

Fig. 4. r.m.s. of the residual distribution between the anodic coordinate of the electron cloud measured by the SDD and the reference anodic coordinate given by the telescope as a function of the drift distance, without any correction (open circles) and applying corrections of the systematic deviations (close circles). The contribution of the microstrip telescopes to the resolution has not been deconvoluted.

Applying the correction of the systematic deviation, an evident improvement of the resolution was obtained, in particular for the large drift distances. It is important to stress the fact that the systematic deviation map was built using the same set of data that was used to construct the resolution curve. This means that possible systematic errors coming from other sources, not necessarily stable during long periods or depending on the measurement procedure, 
are also corrected.

Since it would be difficult to measure the systematic deviations in the ALICE operating conditions, a preliminary study has been already performed in order to built the correction map using an infrared laser in the laboratory. This study has been done on another large area SDD with a $200 \mu \mathrm{m}$ anode pitch, also used during the beam test. The three anodic resolution curves obtained without correction, and applying the correction maps measured in beam test and by using the laser, are compared in fig. 5. Even though the pro-

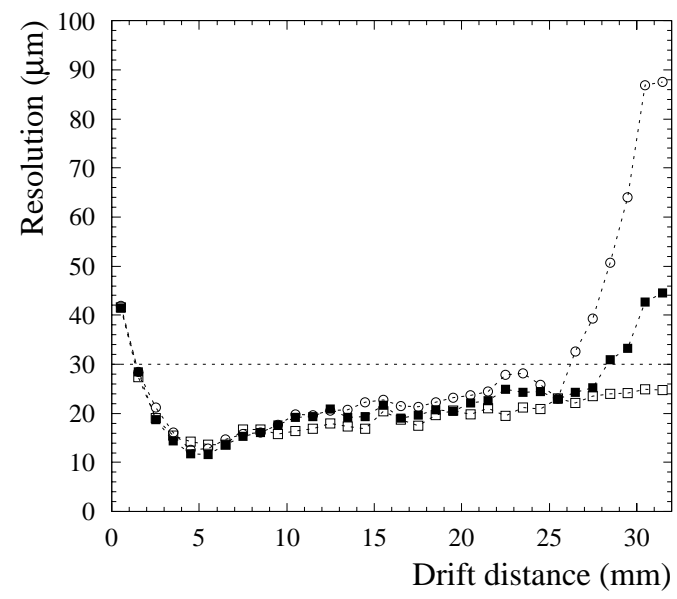

Fig. 5. Anodic resolution of a $200 \mu \mathrm{m}$ anode pitch SDD as a function of the drift distance, without any correction (open circles), applying the corrections measured using the laser (close squares), and applying corrections measured in beam test (open squares).

cedure is still to be optimized, the laboratory setup and the readout system were very different from the beam test experimental conditions, and several months passed between the two measurements, a relevant improvement of the resolution has been obtained using the laboratory measurement.

We wish to thank F. Piuz and P. Martinengo from CERN for the use of the micro-strip telescope and for their assistance during data taking. R.H. wishes to thank Conacyt from Mexico and ICTP from Trieste (Italy) and L.M.M. wishes to thank Conacyt for financial support.

\section{References}

[1] ALICE Collaboration, CERN/LHCC, 95/71

[2] ALICE Collaboration, CERN/LHCC, 99/12

[3] A. Vacchi et al., Nucl. Instr. and Meth. A306 (1991) 187 
[4] A. Castoldi et al., J. Appl. Phys. 71 (7) (1992) 3593

[5] A. Rashevsky et al., these proceedings

[6] W. Dabrowski et al, Nucl. Phys. B 44 (1995) 637

[7] W. von Ammon and H. Herzer, Nucl. Instr. and Meth. 226 (1984) 94

[8] S. Beolè et al., Nucl. Instr. and Meth. A360 (1995) 67-70

[9] S. Beolè et al., submitted to Nucl. Instr. and Meth. 